\title{
Gravity-darkening exponents in semi-detached binary systems from their photometric observations. II.
}

\author{
G. Djurašević ${ }^{1,2}$, H. Rovithis-Livaniou ${ }^{3}$, P. Rovithis ${ }^{4}$, N. Georgiades ${ }^{3}$, S. Erkapić $^{1,2}$, and R. Pavlović ${ }^{1}$ \\ 1 Astronomical Observatory, Volgina 7, 11160 Belgrade, Serbia and Montenegro \\ e-mail: gdjurasevic@aob.bg.ac.yu \\ 2 Isaac Newton Institute of Chile, Yugoslav branch \\ 3 Section of Astrophysics-Astronomy \& Mechanics, Dept. of Physics, Athens University, GR Zografos 157 84, Athens, Greece \\ e-mail: elivan@cc.uoa.gr \\ ${ }^{4}$ Institute of Astronomy \& Astrophysics, National Observatory of Athens, PO Box 20048, 11810 Athens, Greece
}

Received 19 April 2005 / Accepted 21 July 2005

\section{ABSTRACT}

This second part of our study concerning gravity-darkening presents the results for 8 semi-detached close binary systems. From the light-curve analysis of these systems the exponent of the gravity-darkening (GDE) for the Roche lobe filling components has been empirically derived. The method used for the light-curve analysis is based on Roche geometry, and enables simultaneous estimation of the systems' parameters and the gravity-darkening exponents. Our analysis is restricted to the black-body approximation which can influence in some degree the parameter estimation. The results of our analysis are:

1) For four of the systems, namely: TX UMa, $\beta$ Per, AW Cam and TW Cas, there is a very good agreement between empirically estimated and theoretically predicted values for purely convective envelopes.

2) For the AI Dra system, the estimated value of gravity-darkening exponent is greater, and for UX Her, TW And and XZ Pup lesser than corresponding theoretical predictions, but for all mentioned systems the obtained values of the gravity-darkening exponent are quite close to the theoretically expected values.

3) Our analysis has proved generally that with the correction of the previously estimated mass ratios of the components within some of the analysed systems, the theoretical predictions of the gravity-darkening exponents for stars with convective envelopes are highly reliable. The anomalous values of the GDE found in some earlier studies of these systems can be considered as the consequence of the inappropriate method used to estimate the GDE.

4) The empirical estimations of GDE given in Paper I and in the present study indicate that in the light-curve analysis one can apply the recent theoretical predictions of GDE with high confidence for stars with both convective and radiative envelopes.

Key words. stars: binaries: eclipsing - stars: binaries: close - stars: horizontal-branch

\section{Introduction}

In a previous paper (Djurašević et al. 2003, hereafter refer as Paper I), we estimated the gravity-darkening exponents for nine semi-detached binary systems, analyzing their photometric observations. We have continued our study of gravity darkening, and here we present data for a further eight semi-detached binaries.

Ordinary semi-detached systems consist of a primary component inside its Roche lobe and a subgiant (or giant) secondary star filling its corresponding lobe. To analyse this kind of system, we used the latest version of a computer programme (Djurašević et al. 2004) based on Roche geometry and the principles arising from the paper by Wilson \& Devinney (1971). More details on the computational procedure can be found in
Paper I. In the next session we point out only the differences between the earlier and present analysis procedure.

\section{Computational procedure}

The present light-curve analysis was carried out using a simple black-body approximation of the stellar atmosphere, while in Paper I BaSeL models were also used. The reason for this choice is to perform the light-curve analysis in the same way. This was necessary since BaSeL models (Lejeune et al. 1997, 1998) could not be used for all of the analyzed systems. In some particular cases observations were not given in the standard UBV system, and in others the light curves were obtained in the Strömgren system. BaSeL fluxes do not exist for these cases. 
Moreover, a non-linear limb-darkening law was used to avoid the possible negative influence of the wrong evaluation of limb-darkening coefficients on other parameters in the inverse problem. In agreement with Claret (2000b), whose tables we used, we chose the new approximation which can be written as:

$$
\frac{I(\mu)}{I(1)}=1-a_{1}\left(1-\mu^{1 / 2}\right)-a_{2}(1-\mu)-a_{3}\left(1-\mu^{3 / 2}\right)-a_{4}\left(1-\mu^{2}\right)
$$

where $a_{1,2,3,4}$ are the limb-darkening passband specific coefficients and $\mu=\cos \gamma . I(1)$ is the passband specific intensity at the center of the stellar disc, and $\gamma$ is the angle between the line of sight and the emergent flux. This law, based on the leastsquares method, is able to describe the intensity distribution over the whole stellar disk very well, with very good flux conservations. Since the gravity-darkening is a second-order parameter, the correct treatment of the limb-darkening is very important. For a given metallicity, the values of the passband limb-darkening coefficients are derived from the current values of the stellar effective temperature $T_{\text {eff }}$ and surface gravity $\log g$ in each iteration, by interpolation for both of these quantities in Claret's (2000b) tables. This was achieved by bilinear interpolation (Press et al. 1992).

Since the primary component is deep inside its Roche lobe, the primary's shape is very close to that of a sphere. In such a case, and following Rucinski (1969) and Rafert \& Twigg (1980), the gravity-darkening exponents of primary components, $\beta$, are set at their theoretical values. That is, $\beta=0.25$ for stars in hydrostatic and radiative equilibrium (von Zeipel 1924), and $\beta=0.08$ for those with convective envelopes (Lucy 1967). Concerning their albedos, $A$, the values of $A=1.0$ and $A=0.5$ were given.

The reliability of the estimated parameters depends on the quality of photometric observations. This forced us to pay special attention to the choice of the systems. The lack of good observations and radial-velocity measurements of semi-detached systems present a big problem in this type of analysis. We chose only systems with well defined light curves, i.e. systems whose light curves are free of asymmetries and deformations caused by component activity and where all orbital phases are relatively well covered.

\section{Results of the light-curve analysis}

Here we present the results derived from the light-curve analysis of eight semi-detached eclipsing binaries. To estimate the gravity-darkening exponents, we applied the Roche model and the same method of optimisation, enabling a simultaneous evaluation of the parameters of the system and gravity-darkening exponent as in Paper I.

In the tables the first three rows are the total number $n$ of the $B$ and $V$ observations, the final sum of squares of residuals between observed (LCO) and synthetic (LCC) light curves

$$
\sum_{i=1}^{n}\left(\mathrm{O}_{i}-\mathrm{C}_{i}\right)^{2}
$$

and the standard deviation of the observations

$$
\sigma=\sqrt{\frac{\sum_{i=1}^{n}\left(\mathrm{O}_{i}-\mathrm{C}_{i}\right)^{2}}{(n-1)}}
$$

As we pointed in Paper I, the real error bars in the parameter estimates are approximately 2 or 3 times larger than tabular values, which arise from the nonlinear least-squares method applied in the light-curve analysis.

In the figures, the optimum fit of the observed light curves (LCO) to the synthetic ones (LCC) is shown. The $\mathrm{O}-\mathrm{C}$ residuals between the observed (LCO) and optimum synthetic (LCC) light curves are given for each individual light curve.

A view of the Roche model of the systems, obtained with the parameters estimated by analyzing the light curves is also shown, where one can see how each systems looks at a certain orbital phase.

A summary for each system follows.

\subsection{TX Ursae Majoris $\left(P \sim 3^{\mathrm{d}} .063\right)$}

For the analysis of the Algol-type eclipsing binary system TX UMa we used $B$ and $V$ light curves collected by Koch (1961). The temperature of the primary star was taken to be $T_{\mathrm{h}}=12900 \mathrm{~K}$ according to the spectral type of the system (B8V + GIII-IV) as given in Maxted et al. (1995). The gravitydarkening exponent of the primary was fixed to its theoretical value of $\beta_{\mathrm{h}}=0.25$, and the values of the albedos of the components were taken equal to $A_{\mathrm{h}}=1.0$ and $A_{\mathrm{c}}=0.5$. At first the light-curve analysis was made with the spectroscopically estimated mass ratio of the components $q=m_{\mathrm{c}} / m_{\mathrm{h}} \sim 0.248$ (Maxted et al. 1995). The optimum synthetic light curves obtained by solving the inverse problem with this value of the mass ratio were of poor fitting quality. That was very conspicuous around the primary minimum in particular and to some extent around the secondary one. For this reason we reestimated the mass ratio from photometrical observations by employing the q-search method. The so-obtained value of the mass ratio $q=m_{\mathrm{c}} / m_{\mathrm{h}}=0.36$ provided a much better fit of the observations. Also, this re-estimated mass ratio value was in good agreement with the value obtained through simultaneous analysis of light curves and radial velocity curves ( $\left.q=m_{\mathrm{c}} / m_{\mathrm{h}} \sim 0.344\right)$ given by Kang \& Oh (1993).

The parameters derived from the simultaneous light-curve analysis are listed in Table 1 . For the gravity-darkening exponent of the secondary component we found the value $\beta_{\mathrm{c}} \sim 0.076$ which is in very good agreement with the theoretically predicted one for stars with convective envelopes. Figure 1 gives a graphic presentation of these results.

It is evident that with the new photometrically estimated mass ratio $q=m_{\mathrm{c}} / m_{\mathrm{h}}=0.36$ obtained by the q-search method, the optimum model light curves provide a good fit of the observations and the $\mathrm{O}-\mathrm{C}$ residuals are random.

\section{2. $\beta$ Persei $(P \sim 2$. 867$)$}

The two-colour ( $B$ and $V$ ) light curves of Kim (1989) were used to analyse this system. The simultaneous light-curves 

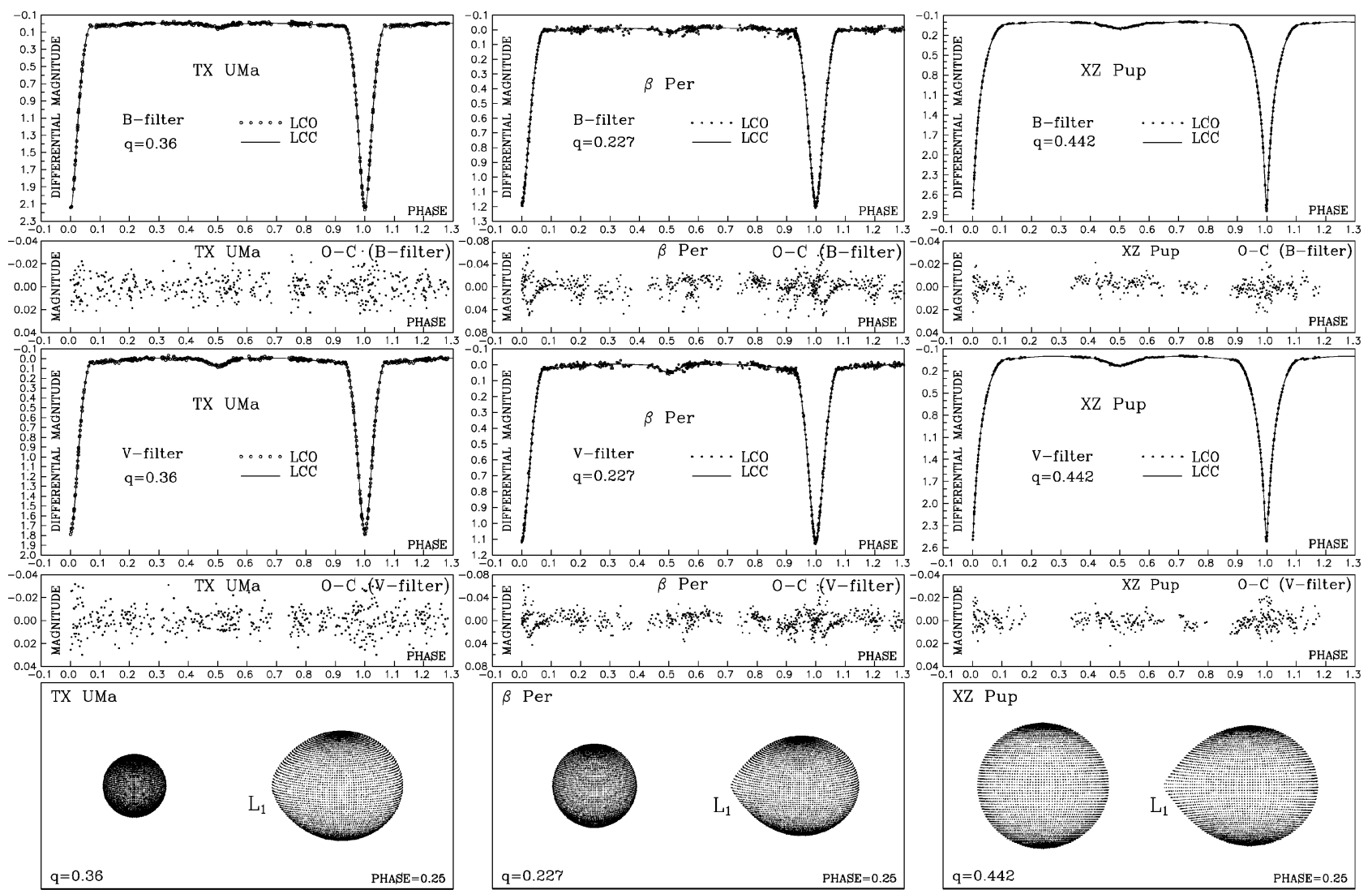

Fig. 1. Observed (LCO) and final synthetic (LCC) light curves of TX UMa, $\beta$ Per and XZ Pup with final O-C residuals and the view of the systems at orbital phase 0.25 , obtained with the parameters estimated by analysing observations.

analysis of $\beta$ Per was made with the photometrically estimated mass ratio of $q=m_{\mathrm{c}} / m_{\mathrm{h}}=0.227$ (Kim 1989). This value is in good agreement with the mass ratio $q=m_{\mathrm{c}} / m_{\mathrm{h}}=$ $0.217 \pm 0.005$ which is obtained from spectroscopic work done by Hill et al. (1971) and Tomkin \& Lambert (1978). The temperature of the primary star was taken to be $T_{\mathrm{h}}=12000 \mathrm{~K}$ (Kim 1989). According to the spectral types of the components (B8 V+K0 IV+A5 V) given in Kim's paper the values of the albedos were fixed to $A_{\mathrm{h}}=1.0$ and $A_{\mathrm{c}}=0.5$, while the gravitydarkening exponent of the primary was set at $\beta_{\mathrm{h}}=0.25$. In the analysis we have also estimated the contribution of the third light to the total light of Algol.

From the results of the light-curve analysis presented in Table 1 and Fig. 1 (second column) we can see that the value of the gravity-darkening exponent for the secondary star, $\beta_{\mathrm{c}}=$ 0.087 , is in very good agreement with the theoretically predicted value for stars with convective envelopes.

For $\beta$ Per by employing a different method Budding \& Kopal (1970) have estimated a large value of the gravitydarkening for the secondary component, which was found to be 3.8 times greater than its theoretical value. Thus, it could be that the anomalous value of the gravity-darkening exponent obtained in this particular case is the consequence of the simplified model not including the effects of the complex physics involved in this kind of systems. Namely, contrary to their result, our analysis give the value of $\beta_{\mathrm{c}} \sim 0.09$ which is in very good agreement with the theoretical prediction for stars with convective envelopes.

In our study we have an essentially different approach based on a modern computer code involving Roche models. It enables real estimates of the optimum parameters of the binary system where model light curves provide a good fit of the observations. Thus, we believe that the anomalous value of the gravity-darkening exponent in Budding \& Kopal's paper should be attributed to the inappropriate method applied.

\section{3. $X Z$ Puppis $(P \sim 2.192)$}

Photoelectric $B$ and $V$ observations of the eclipsing binary XZ Pup published in Johansen et al.'s (1971) paper were used in the present analysis of this system. As the temperature of the hotter primary star we took the value $T_{\mathrm{h}}=9420 \mathrm{~K}$ according to the spectral types of the components $\mathrm{A} 0+\mathrm{G}$, given in Johansen et al.'s (1971) paper and Popper's (1980) calibration. Also, the values of the albedos of the components were taken equal to $A_{\mathrm{h}}=1.0$ and $A_{\mathrm{c}}=0.5$, and the gravity-darkening exponent of the primary was fixed to $\beta_{\mathrm{h}}=0.25$. The mass ratio was estimated from photometrical observations with the q-search method. The so obtained value $q=m_{\mathrm{c}} / m_{\mathrm{h}}=0.442$ provides a better fit of the observations than Budding's (1985) value $\left(q=m_{\mathrm{c}} / m_{\mathrm{h}}=0.43\right)$.

The results obtained from our analysis are given in Table 1 and Fig. 1 (third column). The simultaneous analysis of the $B$ 
Table 1. Results of the analysis of TX UMa, $\beta$ Per and XZ Pup $B$ and $V$ light curves obtained by solving the inverse problem for the Roche model. Gravity-darkening exponent of the cooler secondary component $\left(\beta_{\mathrm{c}}\right)$ is a free parameter.

\begin{tabular}{|c|c|c|c|}
\hline Quantity & TX UMa & $\beta$ Per & XZ Pup \\
\hline$n$ & 730 & 1035 & 579 \\
\hline$\Sigma(\mathrm{O}-\mathrm{C})^{2}$ & 0.0647 & 0.2422 & 0.0245 \\
\hline$\sigma$ & 0.0094 & 0.0153 & 0.0065 \\
\hline$q=m_{\mathrm{c}} / m_{\mathrm{h}}$ & $0.36 \pm 0.01$ & 0.227 & $0.442 \pm 0.006$ \\
\hline$T_{\mathrm{h}}$ & 12900 & 12000 & 9420 \\
\hline$\beta_{\mathrm{h}}$ & 0.25 & 0.25 & 0.25 \\
\hline $\mathrm{A}_{\mathrm{h}}$ & 1.0 & 1.0 & 1.0 \\
\hline$A_{c}$ & 0.5 & 0.5 & 0.5 \\
\hline$f_{\mathrm{h}}=f_{\mathrm{c}}$ & 1.0 & 1.0 & 1.0 \\
\hline$T_{\mathrm{c}}$ & $5742 \pm 13$ & $5323 \pm 21$ & $4991 \pm 14$ \\
\hline$F_{\mathrm{h}}$ & $0.344 \pm 0.001$ & $0.418 \pm 0.001$ & $0.715 \pm 0.001$ \\
\hline$F_{\mathrm{c}}$ & $0.965 \pm 0.001$ & $1.000 \pm 0.001$ & $1.000 \pm 0.001$ \\
\hline$i\left[^{\circ}\right]$ & $81.75 \pm 0.01$ & $82.17 \pm 0.02$ & $89.34 \pm 0.02$ \\
\hline $\mathrm{L}_{3}$ & 0 & $0.104 \pm 0.001$ & 0 \\
\hline$\beta_{\mathrm{c}}$ & $0.076 \pm 0.004$ & $0.087 \pm 0.014$ & $0.06 \pm 0.01$ \\
\hline$a_{1}^{\mathrm{h}, \mathrm{c}}[B]$ & $+0.6195,+0.6647$ & $+0.4561,+0.6454$ & $+0.3807,+0.6027$ \\
\hline$a_{2}^{\mathrm{h}, \mathrm{c}}[B]$ & $+0.3390,-0.0796$ & $+0.7774,+0.0304$ & $+0.9543,-0.7978$ \\
\hline$a_{3}^{\mathrm{h}, \mathrm{c}}[B]$ & $-0.4514,+0.0155$ & $-0.8595,-0.0844$ & $-0.8270,+1.7592$ \\
\hline$a_{4}^{\mathrm{h}, \mathrm{c}}[B]$ & $+0.1659,-0.0081$ & $+0.3091,+0.0261$ & $+0.2545,-0.6388$ \\
\hline$a_{1}^{\mathrm{h}, \mathrm{c}}[V]$ & $+0.3773,+0.4597$ & $+0.4358,+0.5282$ & $+0.5313,+0.6636$ \\
\hline$a_{2}^{\mathrm{h}, \mathrm{c}}[V]$ & $+0.1892,+0.2646$ & $-0.1913,-0.1113$ & $+0.3568,-0.6949$ \\
\hline$a_{3}^{\mathrm{h}, \mathrm{c}}[V]$ & $+0.5787,+0.2689$ & $+1.0456,+0.8113$ & $-0.2767,+1.5718$ \\
\hline$a_{4}^{\mathrm{h}, \mathrm{c}}[V]$ & $-0.2781,-0.1967$ & $-0.4001,-0.3997$ & $+0.0698,-0.6750$ \\
\hline$\Omega_{\mathrm{h}}$ & 6.965 & 5.230 & 3.719 \\
\hline$\Omega_{\mathrm{c}}$ & 2.652 & 2.299 & 2.762 \\
\hline$R_{\mathrm{h}}[D=1]$ & 0.151 & 0.200 & 0.303 \\
\hline$R_{\mathrm{c}}[D=1]$ & 0.266 & 0.241 & 0.290 \\
\hline$L_{\mathrm{h}} /\left(L_{\mathrm{h}}+L_{\mathrm{c}}+L_{3}\right)$ & $0.871[B], 0.842[V]$ & $0.844[B], 0.830[V]$ & $0.946[B], 0.932[V]$ \\
\hline $\mathcal{M}_{\mathrm{h}}\left[\mathcal{M}_{\odot}\right]$ & $4.40 \pm 0.17$ & $1.00 \pm 0.06$ & $2.25 \pm 0.05$ \\
\hline $\mathcal{M}_{\mathrm{c}}\left[\mathcal{M}_{\odot}\right]$ & $1.60 \pm 0.04$ & $0.23 \pm 0.02$ & $0.99 \pm 0.03$ \\
\hline $\mathcal{R}_{\mathrm{h}}\left[\mathcal{R}_{\odot}\right]$ & $2.44 \pm 0.05$ & $2.83 \pm 0.04$ & $3.25 \pm 0.03$ \\
\hline $\mathcal{R}_{\mathrm{c}}\left[\mathcal{R}_{\odot}\right]$ & $4.54 \pm 0.08$ & $3.66 \pm 0.07$ & $3.26 \pm 0.04$ \\
\hline $\log g_{\mathrm{h}}$ & $4.30 \pm 0.02$ & $3.54 \pm 0.02$ & $3.77 \pm 0.02$ \\
\hline $\log g_{\mathrm{c}}$ & $3.33 \pm 0.02$ & $2.67 \pm 0.02$ & $3.41 \pm 0.02$ \\
\hline$M_{\mathrm{bol}}^{\mathrm{h}}$ & $-0.64 \pm 0.02$ & $-0.65 \pm 0.02$ & $0.10 \pm 0.02$ \\
\hline$M_{\text {bol }}^{\mathrm{c}}$ & $1.53 \pm 0.06$ & $2.32 \pm 0.05$ & $2.86 \pm 0.04$ \\
\hline$a_{\mathrm{orb}}\left[R_{\odot}\right]$ & $16.1 \pm 0.2$ & $14.1 \pm 0.1$ & $10.5 \pm 0.5$ \\
\hline
\end{tabular}

Note: $n$ - number of observations, $\Sigma(\mathrm{O}-\mathrm{C})^{2}-$ final sum of squares of residuals between observed and synthetic light curves, $\sigma-$ standard deviation of the observations, $q=m_{\mathrm{c}} / m_{\mathrm{h}}$ - mass ratio of the components, $T_{\mathrm{h}, \mathrm{c}}-$ temperature of the hotter primary and cooler secondary, $\beta_{\mathrm{h}, \mathrm{c}}$, $A_{\mathrm{h}, \mathrm{c}}, f_{\mathrm{h}, \mathrm{c}}$ - gravity-darkening exponents, albedos and nonsynchronous rotation coefficients of the components respectively, $F_{\mathrm{h}, \mathrm{c}}-$ filling factors for the critical Roche lobe of the hotter primary and cooler secondary, $i\left[^{\circ}\right]-$ orbit inclination (in arc degrees), $L_{3}-$ third light, $\beta_{\mathrm{c}}-$ gravity darkening exponent of the cooler secondary component, $a_{1}^{\mathrm{h}, \mathrm{c}}, a_{2}^{\mathrm{h}, \mathrm{c}}, a_{3}^{\mathrm{h}, \mathrm{c}}, a_{4}^{\mathrm{h}, \mathrm{c}}-$ nonlinear limb-darkening coefficients of the components (Claret's formula), $\Omega_{\mathrm{h}, \mathrm{c}},-$ dimensionless surface potentials of the components, $R_{\mathrm{h}, \mathrm{c}}$ - polar radii of the components in units of the distance between the component centres, $L_{\mathrm{h}} /\left(L_{\mathrm{h}}+L_{\mathrm{c}}+L_{3}\right)$ - luminosity of the more massive hotter star, $\mathcal{M}_{\mathrm{h}, \mathrm{c}}\left[\mathcal{M}_{\odot}\right], \mathcal{R}_{\mathrm{h}, \mathrm{c}}\left[\mathcal{R}_{\odot}\right]$, - stellar masses and mean radii of stars in solar units, $\log g_{\mathrm{h}, \mathrm{c}}-\log$ arithm (base 10) of the system components effective gravity, $M_{\mathrm{bol}}^{\mathrm{h}, \mathrm{c}}-$ absolute bolometric magnitudes of systems components and $a_{\mathrm{orb}}\left[R_{\odot}\right]$ - orbital semi-major axis in units of the solar radius. 
and $V$ observations gives $\beta_{\mathrm{c}} \sim 0.06$ for the gravity-darkening exponent of the secondary. By solving individual light curves separately we obtain a somewhat higher value of the gravitydarkening, $\beta_{\mathrm{c}} \sim 0.07$. Both values are in quite good agreement with theoretical predictions. The slight difference between the results of a simultaneous and individual analysis of $B$ and $V$ light curves is expected. Given the lack of a realistic model of the atmosphere, the expected differences between the individual passbands solutions are compensated for and averaged out in the simultaneous analysis of the light curves.

\subsection{TW Andromedae ( $P \sim 4.123)$}

In the analysis of the TW And binary system we have used $B$ and $V$ light curves by Amman \& Walter (1973). With the spectral type F0 V (Hiltner et al. 1949) the temperature of the primary hotter component was equal to $T_{\mathrm{h}}=7200 \mathrm{~K}$, the albedos of the components were fixed to $A_{\mathrm{h}}=1.0$ and $A_{\mathrm{c}}=0.5$, and the gravity-darkening exponent of the primary component was taken to be $\beta_{\mathrm{h}}=0.25$. From Popper's (1989) radial velocity analysis we took the value of the mass ratio for the components $q=m_{\mathrm{c}} / m_{\mathrm{h}}=0.1928$. The same parameters were given in Vivekananda Rao \& Sarma (1997).

Table 2 and Fig. 2 (left column) give the numerical and graphical presentation of the results obtained from the lightcurve analysis of this system. For the gravity-darkening exponent of the secondary component we found the value $\beta_{\mathrm{c}} \sim 0.06$, which is lesser than, but relatively close to the expected one for stars with convective envelopes.

\subsection{TW Cassiopeiae $(P \sim 1 \stackrel{\mathrm{d}}{4} 28)$}

To analyse the TW Cas system we have used McCook's (1971) photoelectric observations. Based on a B9 V spectral type (Struve 1950) the temperature of the hotter primary was fixed to $T_{\mathrm{h}}=10500 \mathrm{~K}$ as in the paper by Narita et al. (2001). According to the spectral types of the stars the albedos were taken to be equal to $A_{\mathrm{h}}=1.0$ and $A_{\mathrm{c}}=0.5$, and the gravitydarkening exponent of the primary component was taken to be $\beta_{\mathrm{h}}=0.25$. Following the same paper we fixed the mass ratio of the components to $q=m_{\mathrm{c}} / m_{\mathrm{h}}=0.432$.

The results of our analysis are given in Table 2 and graphically presented in Fig. 2 (right column). This figure show that with estimated optimum physical and orbital parameters of this system, model light curves provide a very good fit to the observations.

The value of the gravity-darkening exponent for the cooler secondary component is found to be $\beta_{\mathrm{c}} \sim 0.105$ which is greater than, but quite close to the theoretically predicted value for stars with convective envelopes.

\subsection{Al Draconis $(P \sim 1 \mathrm{~d}$.199)}

The well-defined ybv light curves in the Strömgren system of AI Dra were taken from Kiss (2002). Our light-curve analysis was made separately for each passband with the fixed, spectroscopically estimated mass ratio of the components $q=m_{\mathrm{c}} / m_{\mathrm{h}}=0.43$ (Khalesseh 1999). The adopted value of the temperature of the primary was $T_{\mathrm{h}}=9800 \mathrm{~K}$ in agreement with its spectral type B9.6 V (Degirmenci et al. 2000). A fixed value of the gravity-darkening exponent for the primary $\left(\beta_{\mathrm{h}}=0.25\right)$ was used, and the adopted values of the albedos of the components were $A_{\mathrm{h}}=1.0$ and $A_{\mathrm{c}}=0.5$.

Table 3 provides the results of our light-curve analysis. The obtained value $\beta_{\mathrm{c}} \sim 0.12$ for the gravity-darkening exponent of the secondary star is larger than, but relatively close to the theoretically expected one for stars with convective envelopes. Figure 3 (left column) gives a graphical presentation of the results. It is evident that the estimated orbital and physical parameters of this system produced model light curves which fitted the observations very well.

\subsection{UX Hercules $(P \sim 1.549)$}

Yellow and blue photoelectric light curves of this system were collected by Gordon \& Kron (1965). The observations do not cover all orbital phases of the light curves, but the minima are well defined. For this reason we use them in our gravitydarkening study. Based on the spectral classification of the components (A2.6+K) given in Lázaro et al. (1997) we adopted $T_{\mathrm{h}}=9000 \mathrm{~K}$ as the temperature of the hotter primary component. The albedos of the components, $A_{\mathrm{h}}=1.0$ and $A_{\mathrm{c}}=0.5$, and the gravity-darkening exponent of the primary, $\beta_{\mathrm{h}}=0.25$, were taken equal to their theoretically expected values according to this spectral classification.

By applying a q-search method we found the mass ratio $q=m_{\mathrm{c}} / m_{\mathrm{h}}=0.248$, which is in good agreement with the value estimated by Lázaro et al. (1997).

From the results of our simultaneous analysis given in Table 3 we can see that the obtained value of the gravitydarkening exponent for the cooler secondary star, $\beta_{\mathrm{c}}=0.06$, is close to the value expected for stars with convective envelopes. In Fig. 3 (right column) we give the graphical presentation of our results, which shows that the optimum model light curves give a good fit to the observations. The solution obtained with the estimated mass ratio of the components $q=m_{\mathrm{c}} / m_{\mathrm{h}}=0.248$ suggests a semi-detached configuration of the systems although the secondary does not fill completely the corresponding Roche lobe.

\subsection{AW Camelopardis $(P \sim 0.771)$}

In the analysis of the semi-detached system AW Cam, we used $B$ and $V$ observations published by Harris (1968). Following the spectral classification of the components (A0 V + F7 III-IV) given in Russo \& Milano (1983) and using Popper's (1980) calibration we adopted the value of $T_{\mathrm{h}}=$ $9420 \mathrm{~K}$ as the temperature of the hotter primary. The albedos were taken equal to $A_{\mathrm{h}}=1.0$ and $A_{\mathrm{c}}=0.5$ and the gravitydarkening exponent of the primary was fixed to $\beta_{\mathrm{h}}=0.25$.

The light-curve analysis of the system AW Cam show a quite interesting situation. With the fixed mass ratio $q=$ $m_{\mathrm{c}} / m_{\mathrm{h}}=0.22$ (Russo \& Milano 1983) a simultaneous analysis of the $B$ and $V$ light curves gives a relatively good fit to 
Table 2. Results of the analysis of TW And, TW Cas $B$ and $V$ light curves obtained by solving the inverse problem for the Roche model. Gravity-darkening exponent of the cooler secondary component $\left(\beta_{\mathrm{c}}\right)$ is a free parameter.

\begin{tabular}{|c|c|c|c|c|}
\hline CB System & TW And & TW And & TW Cas & TW Cas \\
\hline Quantity & $B$ - filter & $V-$ filter & $B-$ filter & $V-$ filter \\
\hline$n$ & 243 & 246 & 646 & 648 \\
\hline$\Sigma(\mathrm{O}-\mathrm{C})^{2}$ & 0.0268 & 0.0249 & 0.0654 & 0.0516 \\
\hline$\sigma$ & 0.0105 & 0.0101 & 0.0101 & 0.0089 \\
\hline$q=m_{\mathrm{c}} / m_{\mathrm{h}}$ & 0.1928 & & 0.432 & \\
\hline$T_{\mathrm{h}}$ & 7200 & & 10500 & \\
\hline$\beta_{\mathrm{h}}$ & 0.25 & & 0.25 & \\
\hline$A_{\mathrm{h}}$ & 1.0 & & 1.0 & \\
\hline$A_{\mathrm{c}}$ & 0.5 & & 0.5 & \\
\hline$f_{\mathrm{h}}=f_{\mathrm{c}}$ & 1.0 & & 1.0 & \\
\hline$T_{\mathrm{c}}$ & $4366 \pm 14$ & $4464 \pm 15$ & $5408 \pm 39$ & $5497 \pm 25$ \\
\hline$F_{\mathrm{h}}$ & $0.320 \pm 0.001$ & $0.319 \pm 0.001$ & $0.578 \pm 0.003$ & $0.578 \pm 0.003$ \\
\hline$F_{\mathrm{c}}$ & $1.000 \pm 0.001$ & $1.000 \pm 0.001$ & $0.995 \pm 0.001$ & $0.994 \pm 0.001$ \\
\hline$i\left[^{\circ}\right]$ & $87.00 \pm 0.02$ & $87.00 \pm 0.03$ & $74.91 \pm 0.03$ & $74.91 \pm 0.03$ \\
\hline$\beta_{\mathrm{c}}$ & $0.06 \pm 0.01$ & $0.06 \pm 0.01$ & $0.10 \pm 0.02$ & $0.11 \pm 0.01$ \\
\hline$a_{1}^{\mathrm{h}, \mathrm{c}}$ & $+0.3242,+0.5260$ & $+0.3771,+0.6894$ & $+0.5185,+0.5364$ & $+0.5925,+0.5735$ \\
\hline$a_{2}^{\mathrm{h}, \mathrm{c}}$ & $+1.0359,-0.6406$ & $+0.9580,-0.8796$ & $+0.6715,-0.4736$ & $+0.2187,-0.2481$ \\
\hline$a_{3}^{\mathrm{h}, \mathrm{c}}$ & $-0.7923,+1.3181$ & $-0.9534,+1.7085$ & $-0.6770,+1.4342$ & $-0.2243,+0.9946$ \\
\hline$a_{4}^{\mathrm{h}, \mathrm{c}}$ & $+0.2445,-0.2595$ & $+0.3459,-0.6168$ & $+0.2246,-0.5987$ & $+0.0669,-0.4969$ \\
\hline$\Omega_{\mathrm{h}}$ & 6.578 & 6.599 & 4.477 & 4.477 \\
\hline$\Omega_{\mathrm{c}}$ & 2.215 & 2.215 & 2.750 & 2.752 \\
\hline$R_{\mathrm{h}}[D=1]$ & 0.157 & 0.156 & 0.246 & 0.246 \\
\hline$R_{\mathrm{c}}[D=1]$ & 0.230 & 0.230 & 0.287 & 0.287 \\
\hline$L_{\mathrm{h}} /\left(L_{\mathrm{h}}+L_{\mathrm{c}}\right)$ & 0.874 & 0.786 & 0.917 & 0.863 \\
\hline $\mathcal{M}_{\mathrm{h}}\left[M_{\odot}\right]$ & $1.68 \pm 0.07$ & & $2.66 \pm 0.08$ & \\
\hline $\mathcal{M}_{\mathrm{c}}\left[M_{\odot}\right]$ & $0.32 \pm 0.02$ & & $1.15 \pm 0.05$ & \\
\hline $\mathcal{R}_{\mathrm{h}}\left[R_{\odot}\right]$ & $2.14 \pm 0.05$ & & $2.07 \pm 0.08$ & \\
\hline $\mathcal{R}_{\mathrm{c}}\left[R_{\odot}\right]$ & $3.38 \pm 0.09$ & & $2.55 \pm 0.07$ & \\
\hline $\log g_{\mathrm{h}}$ & $4.01 \pm 0.02$ & & $4.23 \pm 0.02$ & \\
\hline $\log g_{\mathrm{c}}$ & $2.89 \pm 0.02$ & & $3.68 \pm 0.02$ & \\
\hline$M_{\mathrm{bol}}^{\mathrm{h}}$ & $2.18 \pm 0.02$ & & $0.61 \pm 0.03$ & \\
\hline$M_{\text {bol }}^{\mathrm{c}}$ & $3.31 \pm 0.05$ & & $3.03 \pm 0.05$ & \\
\hline$a_{\mathrm{orb}}\left[R_{\odot}\right]$ & $13.64 \pm 0.22$ & & $8.32 \pm 0.20$ & \\
\hline
\end{tabular}

Note: same as in Table 1.

the observations, but it demands an anomalous value of the gravity-darkening exponent for the secondary $\operatorname{star}\left(\beta_{\mathrm{c}} \sim 0.40\right)$. This value is 5 times higher than we expect according to Lucy's (1967) or Claret's (1998) predictions for a star with a convective envelope.

This unexpectedly high value needs an explanation. The light curves do not indicate the presence of any activity of the components that can be used as an explanation of the gravitydarkening anomalies. So, we tried to find a light-curve solution with fixed gravity-darkening exponents corresponding to the theoretical predictions for the components in the system. The Roche model in this case provided a quite good fit of the observations, but it was necessary to revise the estimated value of the mass ratio of the components.

Since there is no spectroscopically estimated mass ratio for this system, we apply the q-search method to estimate this parameter photometrically. The use of the q-search method proved that the optimum fit of the observations was obtained with the mass ratio of $q=m_{\mathrm{c}} / m_{\mathrm{h}}=0.36$. In this case the gravity-darkening exponent of the secondary star had a normal value and the model light curves gave a better fit of the observations. With this value of the mass ratio we lose the semi-detached configuration and that both components are inside their Roche lobes. This solution appears reasonable. 

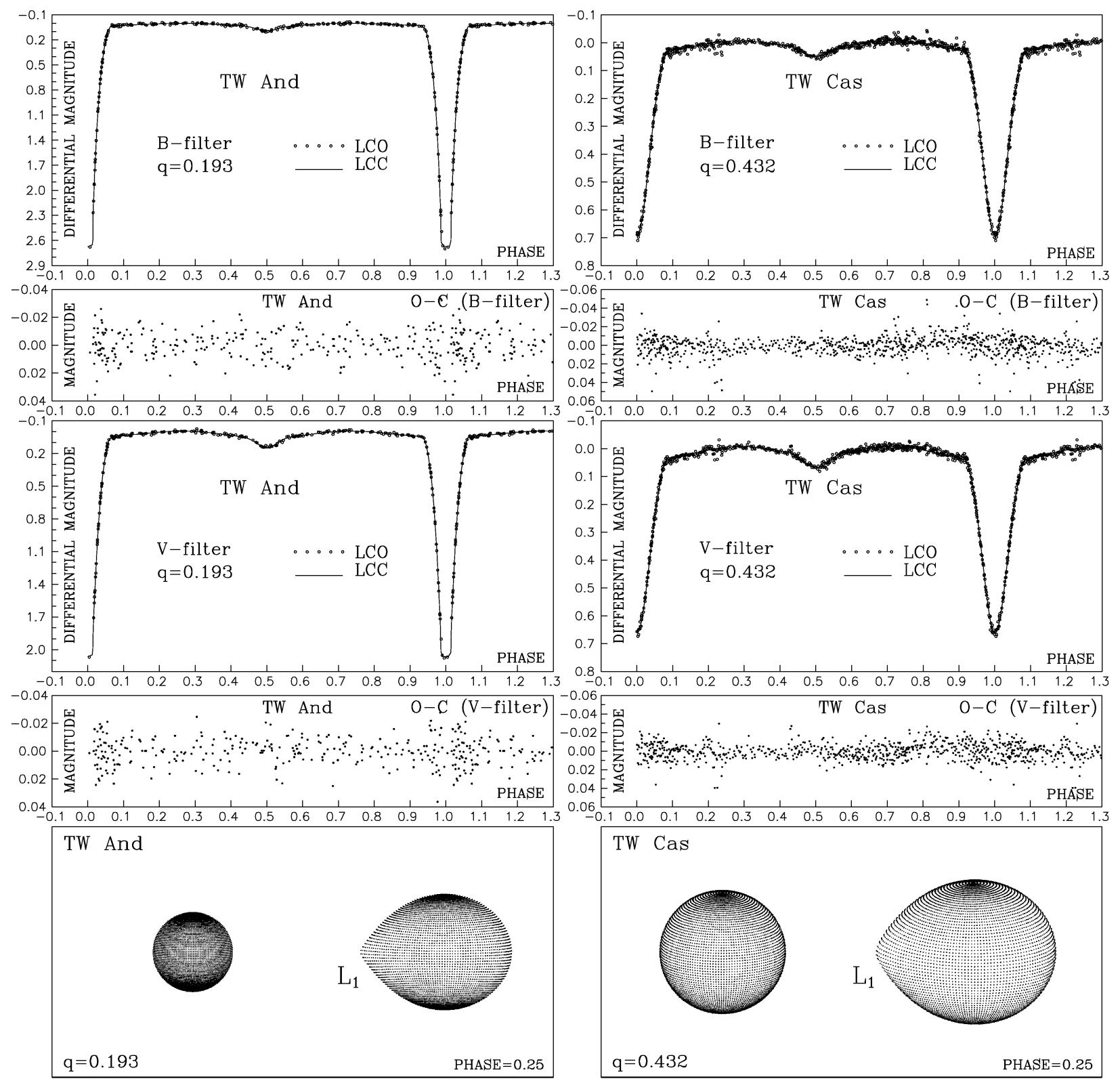

Fig. 2. Observed (LCO) and final synthetic (LCC) light curves of TW And (left) and TW Cas (right) with final O-C residuals and the view of the systems at orbital phase 0.25 , obtained with the parameters estimated by analysing observations.

Russo \& Milano (1983) estimate their value of the mass ratio by assuming a priori the semi-detached configuration. Here we have shown that without introducing this assumption, which limits in advance the use of a Roche model to a particular type of system, by applying a q-search method we obtain a quite different value of the mass ratio. Moreover, the obtained solutions suggest that there is no gravity-darkening anomaly, and that the Roche model provides a better fit to the observations. We believe that this solution is more acceptable. The final solution of this problem can be provided by radial velocity measurements of the components that can give a real estimate of the mass ratio and consequently a reliable estimate of the gravity-darkening exponent.

Table 4 shows the results of our analysis, and these are graphically presented in Fig. 4. The left sides of the Table 4 and Fig. 4 present the solution with Russo \& Milano's (1983) value of the mass ratio, and the right sides show the results with the new mass ratio, obtained with the q-search.

\section{Discussion and conclusions}

In Paper I, nine semi-detached systems had been analyzed; some of these had either one or both of their components of very early spectral type. In the present study the secondary components of all 8 systems are stars with convective envelopes.

The light curve analyses of eight semi-detached binary systems, presented in this paper, were made within the Roche geometry, allowing us to estimate the gravity-darkening exponents of the secondary components filling their Roche lobes. 
Table 3. The results of analysis of AI Dra Strömgren $y b v$ light curves and UX Her $B$ and $V$ light curves obtained by solving the inverse problem for the Roche model. The gravity-darkening exponent of the cooler secondary component $\left(\beta_{\mathrm{c}}\right)$ is a free parameter.

\begin{tabular}{|c|c|c|c|c|}
\hline $\begin{array}{l}\text { CB System } \\
\text { Quantity } \\
\end{array}$ & $\begin{array}{l}\text { AI Dra } \\
y \text { - filter }\end{array}$ & $\begin{array}{l}\text { AI Dra } \\
b-\text { filter }\end{array}$ & $\begin{array}{l}\text { AI Dra } \\
v-\text { filter }\end{array}$ & $\begin{array}{l}\text { UX Her } \\
B, V-\text { filtrs }\end{array}$ \\
\hline $\bar{n}$ & 2635 & 2635 & 2635 & 696 \\
\hline$\Sigma(\mathrm{O}-\mathrm{C})^{2}$ & 0.0862 & 0.0927 & 0.1213 & 0.0344 \\
\hline$\sigma$ & 0.0057 & 0.0059 & 0.0068 & 0.0071 \\
\hline$q=m_{\mathrm{c}} / m_{\mathrm{h}}$ & 0.43 & & & $0.248 \pm 0.007$ \\
\hline$T_{\mathrm{h}}$ & 9800 & & & 9000 \\
\hline$\beta_{\mathrm{h}}$ & 0.25 & & & 0.25 \\
\hline$A_{\mathrm{h}}$ & 1.0 & & & 1.0 \\
\hline$A_{\mathrm{c}}$ & 0.5 & & & 0.5 \\
\hline$f_{\mathrm{h}}=f_{\mathrm{c}}$ & 1.0 & & & 1.0 \\
\hline$T_{\mathrm{c}}$ & $5607 \pm 15$ & $5550 \pm 16$ & $5445 \pm 20$ & $4055 \pm 22$ \\
\hline$F_{\mathrm{h}}$ & $0.670 \pm 0.001$ & $0.669 \pm 0.001$ & $0.670 \pm 0.00$ & $0.483 \pm 0.001$ \\
\hline$F_{\mathrm{c}}$ & $0.990 \pm 0.001$ & $0.992 \pm 0.001$ & $0.993 \pm 0.001$ & $0.931 \pm 0.001$ \\
\hline$i\left[^{\circ}\right]$ & $77.40 \pm 0.08$ & $77.37 \pm 0.08$ & $77.42 \pm 0.08$ & $82.12 \pm 0.01$ \\
\hline$\beta_{\mathrm{c}}$ & $0.120 \pm 0.007$ & $0.123 \pm 0.007$ & $0.118 \pm 0.009$ & $0.06 \pm 0.02$ \\
\hline$a_{1}^{\mathrm{h}, \mathrm{c}}$ & $+0.5543,+0.5400$ & $+0.4513,+0.4155$ & $+0.4390,+0.5413$ & $+0.4027,+0.5209[B] ;+0.5877,+0.7136[V]$ \\
\hline$a_{2}^{\mathrm{h}, \mathrm{c}}$ & $+0.3222,-0.1002$ & $+0.8004,+0.0291$ & $+0.8980,-0.6903$ & $+0.7907,+0.3053[B] ;-0.6993,-0.9308[V]$ \\
\hline$a_{3}^{\mathrm{h}, \mathrm{c}}$ & $-0.2696,+0.8016$ & $-0.7284,+0.8797$ & $-0.8103,+1.7817$ & $-0.5422,-0.1246[B] ;+1.1472,+1.5821[V]$ \\
\hline$a_{4}^{\mathrm{h}, \mathrm{c}}$ & $+0.0717,-0.4229$ & $+0.2283,-0.4501$ & $+0.2534,-0.7090$ & $+0.1238,-0.0076[B] ;-0.0969,-0.4541[V]$ \\
\hline$\Omega_{\mathrm{h}}$ & 3.911 & 3.915 & 3.910 & 4.641 \\
\hline$\Omega_{\mathrm{c}}$ & 2.755 & 2.751 & 2.750 & 2.427 \\
\hline$R_{\mathrm{h}}[D=1]$ & 0.286 & 0.286 & 0.286 & 0.227 \\
\hline$R_{\mathrm{c}}[D=1]$ & 0.285 & 0.286 & 0.286 & 0.231 \\
\hline$L_{\mathrm{h}} /\left(L_{\mathrm{h}}+L_{\mathrm{c}}\right)$ & 0.867 & 0.902 & 0.933 & $0.981[B] ; 0.973[V]$ \\
\hline $\mathcal{M}_{\mathrm{h}}\left[M_{\odot}\right]$ & $2.79 \pm 0.02$ & & & $2.28 \pm 0.09$ \\
\hline $\mathcal{M}_{\mathrm{c}}\left[M_{\odot}\right]$ & $1.20 \pm 0.02$ & & & $0.56 \pm 0.06$ \\
\hline $\mathcal{R}_{\mathrm{h}}\left[R_{\odot}\right]$ & $2.19 \pm 0.02$ & & & $1.82 \pm 0.04$ \\
\hline $\mathcal{R}_{\mathrm{c}}\left[R_{\odot}\right]$ & $2.30 \pm 0.02$ & & & $1.94 \pm 0.04$ \\
\hline $\log g_{\mathrm{h}}$ & $4.20 \pm 0.02$ & & & $4.27 \pm 0.02$ \\
\hline $\log g_{\mathrm{c}}$ & $3.79 \pm 0.02$ & & & $3.62 \pm 0.02$ \\
\hline$M_{\mathrm{bol}}^{\mathrm{h}}$ & $0.79 \pm 0.02$ & & & $1.56 \pm 0.07$ \\
\hline$M_{\mathrm{bol}}^{\mathrm{c}}$ & $3.17 \pm 0.06$ & & & $4.89 \pm 0.06$ \\
\hline$a_{\mathrm{orb}}\left[R_{\odot}\right]$ & $7.52 \pm 0.01$ & & & $7.97 \pm 0.02$ \\
\hline
\end{tabular}

Note: Same as in Table 1.

The gravity-darkening exponents of the components underfilling their Roche lobes were fixed to their theoretical values. That is to $\beta=0.25$ for stars in hydrostatic and radiative equilibrium, according to von Zeipel's (1924) value, and to $\beta=0.08$ for stars with convective envelopes, according to Lucy's (1967) calculations.

The light-curve analysis of these systems gave us new estimates for their physical and orbital parameters and the obtained fit of the observations is good.

The absolute parameters of the systems given in the tables are derived from the light-curve solutions, while the mass ratio of the components and semi-major orbital axis are estimated from corresponding radial velocity solutions. For some of the analysed systems with a previously photometrically estimated mass ratio either we could not obtain a good fit to the observations or the obtained values of the gravity-darkening exponent were anomalous. Therefore, we re-estimated this parameter by analysing the light curves with the q-search method. The obtained photometric mass ratio of the components improved the quality of the fit and the agreement between theoretical and quasi-empirical estimates of gravity-darkening exponents.

For systems where we do not have the data on radial velocities, a rough estimate of the semi-major axis is obtained from the orbital period of the system and from appropriate estimates of the masses of the components found in the given references.

According to the old theoretical predictions of Lucy (1967) the expected gravity-darkening exponents should be 0.08 . The final derived values, as one can see from Tables $1-4$ are in the 

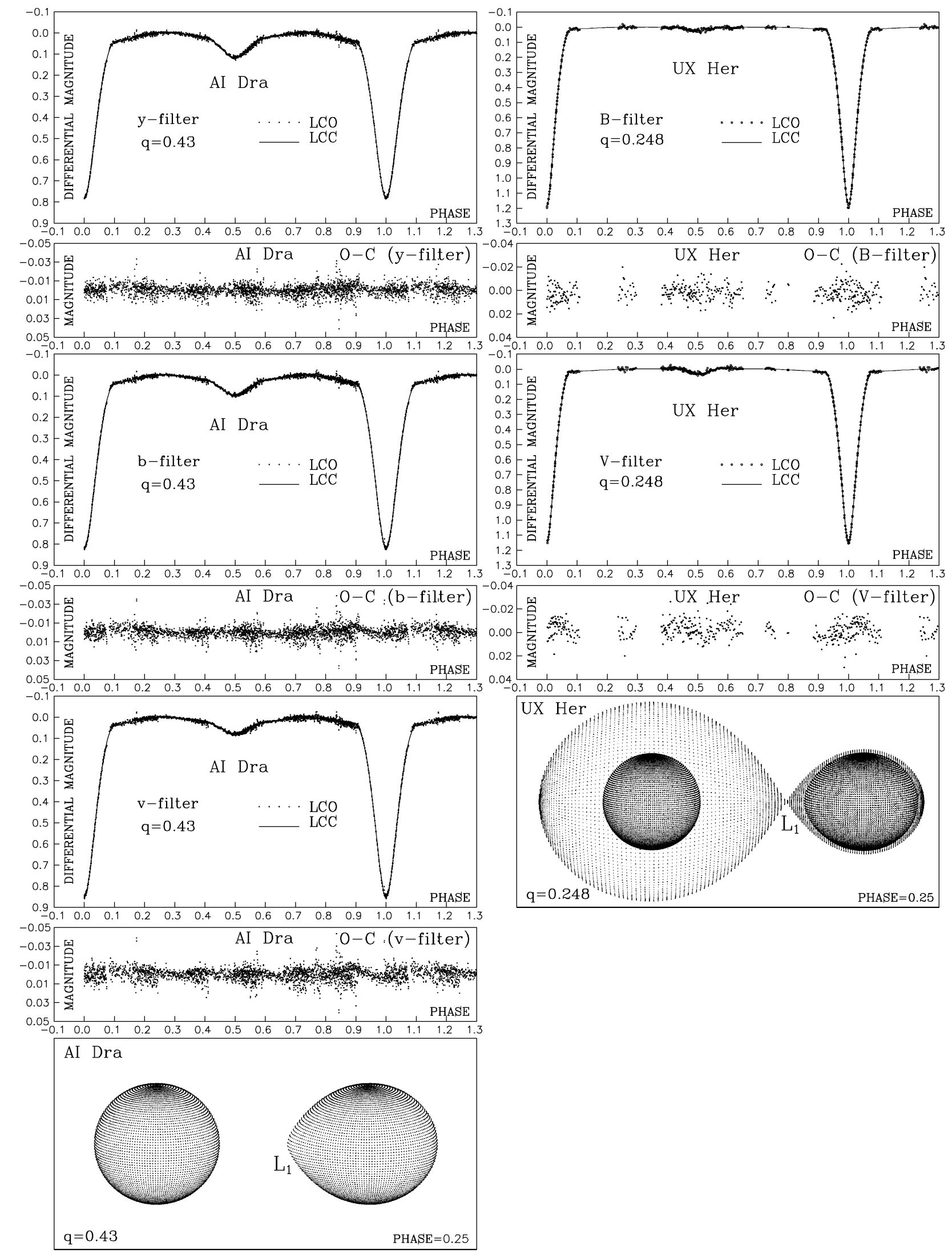

Fig. 3. Observed (LCO) and final synthetic (LCC) light curves of AI Dra and UX Her with final O-C residuals and the view of the systems at orbital phase 0.25 , obtained with the parameters estimated by analysing observations. 
Table 4. Simultaneous analysis of AW Cam $B$ and $V$ light curves obtained by solving the inverse problem for the Roche model with fixed and photometrically estimated mass ratios. The gravity-darkening exponent of the cooler secondary component $\left(\beta_{\mathrm{c}}\right)$ is a free parameter.

\begin{tabular}{|c|c|c|}
\hline Quantity & $q-$ fixed (Russo \& Milano, 1983) & $q$-photometrically estimated \\
\hline$n$ & 1463 & 1463 \\
\hline$\Sigma(\mathrm{O}-\mathrm{C})^{2}$ & 0.1415 & 0.1184 \\
\hline$\sigma$ & 0.0098 & 0.0090 \\
\hline$q=m_{\mathrm{c}} / m_{\mathrm{h}}$ & 0.22 & $0.36 \pm 0.03$ \\
\hline$T_{\mathrm{h}}$ & 9420 & 9420 \\
\hline$\beta_{\mathrm{h}}$ & 0.25 & 0.25 \\
\hline $\mathrm{A}_{\mathrm{h}}$ & 1.0 & 1.0 \\
\hline $\mathrm{A}_{\mathrm{c}}$ & 0.5 & 0.5 \\
\hline$f_{\mathrm{h}}=f_{\mathrm{c}}$ & 1.0 & 1.0 \\
\hline $\mathrm{T}_{\mathrm{c}}$ & $6464 \pm 24$ & $5921 \pm 30$ \\
\hline $\mathrm{F}_{\mathrm{h}}$ & $0.803 \pm 0.002$ & $0.870 \pm 0.001$ \\
\hline $\mathrm{F}_{\mathrm{c}}$ & $0.991 \pm 0.001$ & $0.783 \pm 0.001$ \\
\hline$i\left[^{\circ}\right]$ & $75.28 \pm 0.04$ & $77.67 \pm 0.04$ \\
\hline$\beta_{\mathrm{c}}$ & $0.40 \pm 0.02$ & $0.08 \pm 0.03$ \\
\hline$a_{1}^{\mathrm{h}, \mathrm{c}}$ & $+0.4414,+0.3068[B] ;+0.5412,+0.3883[V]$ & $+0.4371,+0.4342[B] ;+0.5410,+0.4961[V]$ \\
\hline$a_{2}^{\mathrm{h}, \mathrm{c}}$ & $+0.7741,+0.7883[B] ;+0.3032,+0.7388[V]$ & $+0.7885,+0.0814[B] ;+0.3073,+0.1588[V]$ \\
\hline$a_{3}^{\mathrm{h}, \mathrm{c}}$ & $-0.6307,-0.3157[B] ;-0.1997,-0.5231[V]$ & $-0.6471,+0.7226[B] ;-0.2067,+0.3988[V]$ \\
\hline$a_{4}^{\mathrm{h}, \mathrm{c}}$ & $+0.1779,+0.0518[B] ;+0.0353,+0.1475[V]$ & $+0.1845,-0.3737[B] ;+0.0386,-0.2634[V]$ \\
\hline$\Omega_{\mathrm{h}}$ & 2.800 & 2.948 \\
\hline$\Omega_{\mathrm{c}}$ & 2.291 & 2.980 \\
\hline$R_{\mathrm{h}}[D=1]$ & 0.385 & 0.383 \\
\hline$R_{\mathrm{c}}[D=1]$ & 0.237 & 0.215 \\
\hline$L_{\mathrm{h}} /\left(L_{\mathrm{h}}+L_{\mathrm{c}}\right)$ & $0.915[B], 0.906[V]$ & $0.956[B], 0.950[V]$ \\
\hline $\mathcal{M}_{\mathrm{h}}\left[\mathcal{M}_{\odot}\right]$ & $3.20 \pm 0.03$ & $2.87 \pm 0.03$ \\
\hline $\mathcal{M}_{\mathrm{c}}\left[\mathcal{M}_{\odot}\right]$ & $0.71 \pm 0.03$ & $1.04 \pm 0.03$ \\
\hline $\mathcal{R}_{\mathrm{h}}\left[\mathcal{R}_{\odot}\right]$ & $2.22 \pm 0.02$ & $2.22 \pm 0.02$ \\
\hline $\mathcal{R}_{\mathrm{c}}\left[\mathcal{R}_{\odot}\right]$ & $1.42 \pm 0.02$ & $1.23 \pm 0.02$ \\
\hline $\log g_{\mathrm{h}}$ & $4.25 \pm 0.02$ & $4.20 \pm 0.02$ \\
\hline $\log g_{\mathrm{c}}$ & $3.98 \pm 0.02$ & $4.27 \pm 0.02$ \\
\hline$M_{\mathrm{bol}}^{\mathrm{h}}$ & $0.94 \pm 0.03$ & $0.93 \pm 0.03$ \\
\hline$M_{\mathrm{bol}}^{\mathrm{c}}$ & $3.54 \pm 0.06$ & $4.22 \pm 0.06$ \\
\hline$a_{\mathrm{orb}}\left[R_{\odot}\right]$ & $5.57 \pm 0.09$ & $5.57 \pm 0.09$ \\
\hline
\end{tabular}

Note: Same as in Table 1.

range 0.06-0.12, close to Lucy's predicted value for a star with a convective envelope.

The values of the gravity-darkening exponents estimated in the light-curve analysis were also compared to the recent theoretical predictions obtained on the basis of Claret's (1998) tables. By interpolating the data from Claret's tables we obtained the tracks for corresponding observed masses of the secondary system components. These tracks are represented in Fig. 5 together with the semi-empirical values of the GDEs estimated from our light-curve analysis. In certain cases the masses of the secondaries are lower than the lower mass limit of the Claret's (1998) calculations. In these situations we applied extrapolation of the data from Claret's tables. In Fig. 5 GDE for a very low mass star model (Claret 2000a) are represented by the dashed line. The picture in the upper right corner shows the results given in Paper I.

Our results graphically presented in Fig. 5 show that, in general, the estimated values of gravity-darkening exponents are in close agreement with the theoretically predicted tracks for stars with convective envelopes.

Our results for the analysed systems can be summarized as follows:

A. For the systems TX UMa, $\beta$ Per, TW Cas and AW Cam empirically estimated gravity-darkening exponent, obtained in the light-curve analysis are in good agreement with theoretically predicted tracks. 

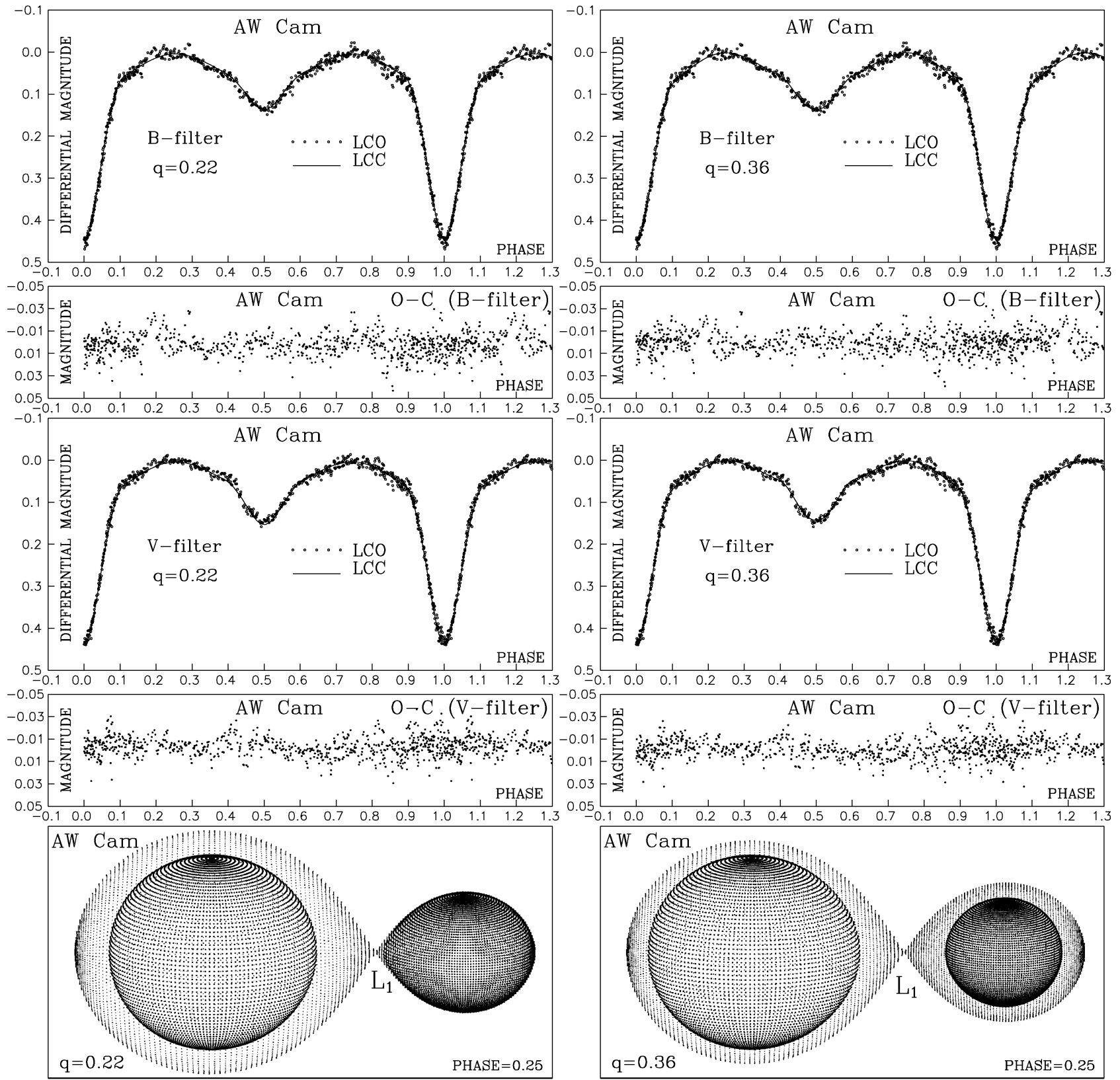

Fig. 4. Observed (LCO) and final synthetic (LCC) light curves of AW Cam with final O-C residuals obtained by solving the inverse problem for the Roche model with old fixed $\left(q=m_{\mathrm{c}} / m_{\mathrm{h}}=0.22\right)$ and new photometrically estimated mass ratio $\left(q=m_{\mathrm{c}} / m_{\mathrm{h}}=0.36\right)$ and the view of the system at orbital phase 0.25 , obtained with the parameters estimated by analysing observations.

For the system of TX UMa, the light-curve analysis proved that with the new photometrically estimated mass ratio $q=$ $m_{\mathrm{c}} / m_{\mathrm{h}}=0.36$ obtained with the q-search method, we have full agreement between empirically estimated values of the gravitydarkening exponent $\left(\beta_{\mathrm{c}} \sim 0.08\right)$ and the theoretically predicted one.

For $\beta$ Per, our analysis gives the value of $\beta_{\mathrm{c}} \sim 0.09$ which is in good agreement with the theoretical predictions for stars with convective envelopes. By employing a different method, Budding \& Kopal (1970) have estimated a large value for the gravity-darkening of the secondary component, which was found to be 3.8 times greater than its theoretical value. We believe that the reason for the anomalous value of the gravity-darkening exponent in their paper can be attributed to the unappropriate method applied.

For the gravity-darkening exponents of the secondary components of TW Cas we found $\beta_{\mathrm{c}} \sim 0.105$.

With the fixed mass ratio of AW Cam $q=m_{\mathrm{c}} / m_{\mathrm{h}}=0.22$ (Russo \& Milano 1983) a simultaneous analysis of the $B$ and $V$ light curves gives a relatively good fit of the observations, but it demands an anomalous value of the gravitydarkening exponent for the secondary star $\left(\beta_{\mathrm{c}} \sim 0.40\right)$. This value is 5 times higher than we can expect according to Lucy's (1967) or Claret's (1998) predictions for a star with a convective envelope. 


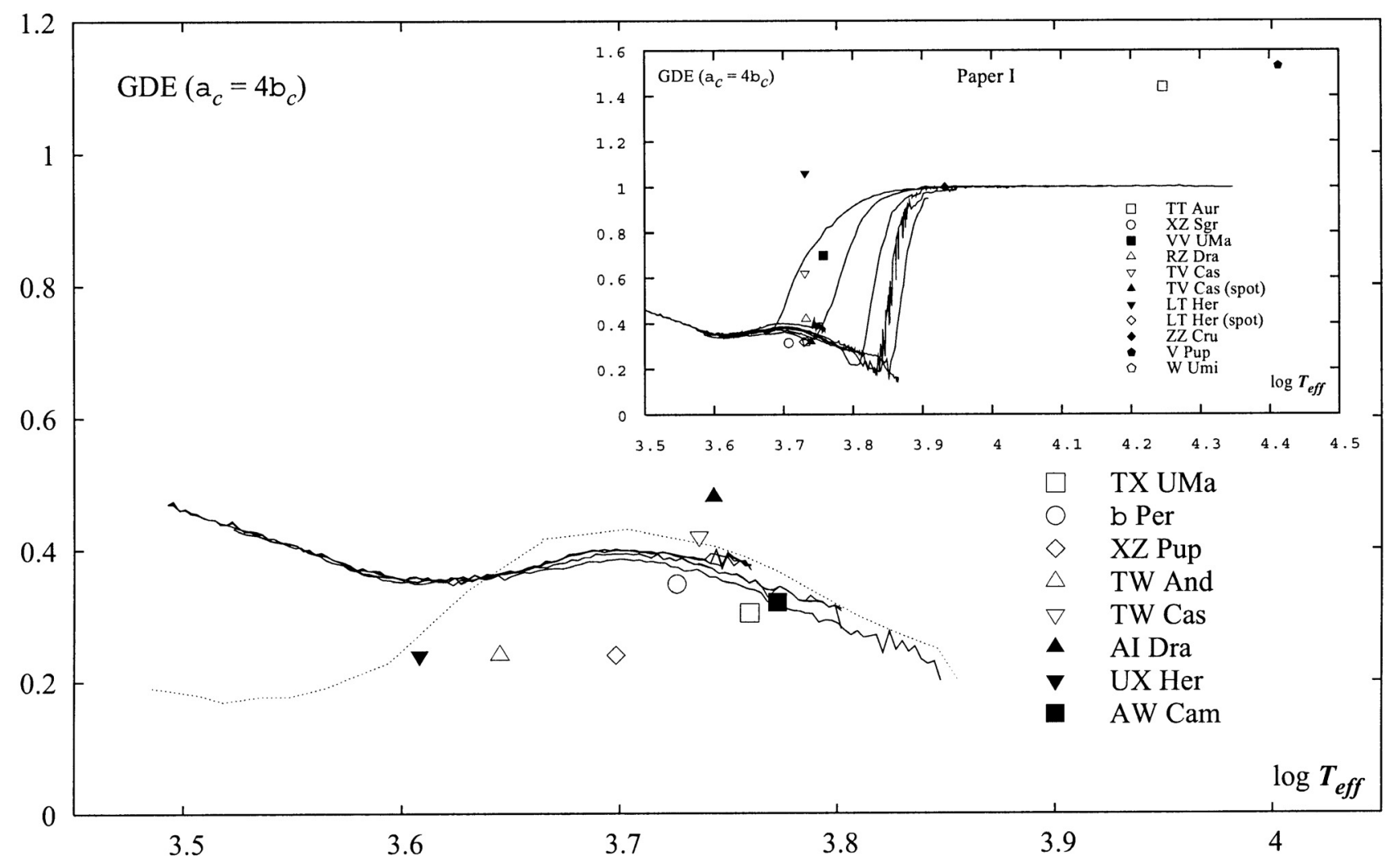

Fig. 5. Empirical gravity-darkening exponent (GDE) values and Claret's (1998) theoretical predictions as a function of effective temperature. The lines represent the tracks for corresponding observed masses of the secondary components. The dashed line represents GDE for a very low mass star model (Claret 2000a). The picture in the upper right corner shows the results given in Paper I.

We have shown that this unexpectedly high value (for which a physically acceptable explanation is difficult to find) can be a consequence of the incorrect evaluation of the mass ratio of the components. Without an a priori assumed semidetached configuration the q-search method proved that the optimum fit of the observations was obtained with the mass ratio of $q=m_{\mathrm{c}} / m_{\mathrm{h}}=0.36$. In this case the gravity-darkening exponent of the secondary star had a normal, theoretically expected value and the model light curves gave a better fit of the observations. This problem can be resolved by radial velocity measurements of the components for a real estimate of the mass ratio.

B. For XZ Pup, using the new photometrically estimated mass ratio of the components $\left(q=m_{\mathrm{c}} / m_{\mathrm{h}}=0.442\right)$, simultaneous analysis of the $B$ and $V$ observations gives $\beta_{\mathrm{c}} \sim 0.06$ for the gravity-darkening exponent of the secondary. The same GDE values have been obtained for TW And and UX Her. This value although somewhat smaller than the theoretically predicted one confirms the validity of theoretical calculations for stars with convective envelopes.

C. On the other hand, for the gravity-darkening exponent of the secondary in the AI Dra system, the present analysis gives the value $\beta_{\mathrm{c}} \sim 0.12$, which is greater, but relatively close to the theoretically predicted track for stars with a convective envelope.

With the revision of the previously estimated mass ratios of the components within some of the analysed systems, our analysis has indicated that in general the theoretical predictions of the gravity-darkening exponent for stars with convective envelopes have high validity. We show that anomalous values found in some earlier studies may be attributed to an inappropriate method applied to estimations of the gravity-darkening exponent.

The discrepancies between the empirically estimated values of the gravity-darkening exponent and theoretical predictions can be partly explained by the insufficient accuracy of the analysed light curves in the estimation of this second order parameter, and by the lack of reliable estimates of the input parameters such as the mass ratio, temperature, real model atmospheres of the components etc. Because of the existing gas stream between the components, the shape of the light curve in semi-detached systems can be also affected by the presence of nonphotospheric matter, which can consequently be reflected in the estimates of the gravity-darkening exponent. Similar effects also can be induced by the activity of the components in the system.

On the other hand, theoretical studies suggest that the gravity-darkening phenomenon is not related only to the atmospheric parameters but it is also affected by the internal stellar structure and by the details of the rotation law (Claret 2000a). The previous values of 0.32 and 1.0 for convective and radiative envelopes are superseded by Claret's (1998) calculations where a smooth transition is achieved between both energy transport mechanisms. Both processes of energy transport can even exist simultaneously in a determined stellar envelope. The influence of the chemical composition on the gravity-darkening 
exponent is rated as slight and located mainly in the zone of the radiative/convective phase transition. Moreover, Claret (2000a) shows that the gravity-darkening exponent depends on the angular velocity distribution, on the opacity law and on the star's differential rotation. His paper suggests differential rotation as a possible cause of the anomalous gravity-darkening exponents within early-type systems. The presence of an accretion disk, detected in some detached systems, may change the behaviour of the gravity-darkening exponent too. Possible explanations for the discrepancies between empirical GDEs and theoretical predictions are suggested in Paper I.

The choice of the black-body approximation limits to some extent the analysis carried out here. For systems where a more realistic approximation of stellar atmosphere radiation was possible (e.g. BaSeL), the tests indicated that the results were insignificantly changed compared to the black-body approximation, affecting above all the temperature of the secondary component and thereby indirectly very slightly affecting other system parameters. Since the impact of the applied approximation of the radiation character on the final results of the light-curve analysis is quite small, we adopt the black-body approximation to keep the consistency of the procedure for all the systems analysed.

Generally, our empirical estimations of GDE given in Paper I and in the present study indicate that in the light-curve analysis one can apply the recent theoretical predictions of GDE with high confidence for stars with both convective and radiative envelopes.

Acknowledgements. This work has been supported by the Athens University, (grant no. 70/4/3305), and by the Ministry for Science and Environmental Protection of Serbia through the project "Stellar and Solar physics". The authors would like to thank Dr Claret for his comments and suggestions that helped to improve the quality of the paper.

\section{References}

Amman, M., \& Walter, K. 1973, A\&A, 24, 131

Budding, E. 1985, PASP, 97, 584

Budding, E., \& Kopal, Z. 1970, Ap\&SS, 9, 343
Claret, A. 1995, A\&AS, 109, 441

Claret, A. 1998, A\&AS, 131, 395

Claret, A. 2000a, A\&A, 359, 289

Claret, A. 2000b, A\&A, 363, 1081

Degirmenci, Ö. L., Gülmen, Ö., Sezer, C., et al. 2000, A\&A, 363, 244

Djurašević, G., Rovithis-Livaniou, H., Rovithis, P., et al. 2003, A\&A, 402, 667

Djurašević, G., Albayrak, B., Selam, S. O., Erkapić, S., \& Şenavc1, H. V. 2004, New Astronomy, 9, 425

Gordon, K. C., \& Kron, G. E. 1965, AJ, 70, 100

Harris, A. J. 1968, AJ, 73, 164

Hill, G., Barnes, J. V., Hutchings, J. B., \& Pearce, J. A. 1971, ApJ, 168,433

Hiltner, W. A., Smith, B., \& Struve, O. 1949, ApJ, 109, 95

Johansen, K. T., Jörgensen, H. E., \& Bohr, V. 1971, A\&A, 11, 20

Kang, Y. W., \& Oh, K. D. 1993, Ap\&SS, 201, 177

Khalesseh, B. 1999, Ap\&SS, 260, 299

Kim, H.-I. 1989, ApJ, 342, 1061

Kiss, L. L. 2002, IBVS 5355

Koch, R. H. 1961, AJ, 66, 230

Lázaro, C., Martınez-Pais, I. G., \& Arévalo, M. J. 1997, AJ, 113, 1122

Lejeune, T., Cuisinier, F., \& Buser, R. 1997, A\&AS, 125, 229

Lejeune, T., Cuisinier, F., \& Buser, R. 1998, A\&AS, 130, 65

Lucy, L. B. 1967, Zs. f. Ap., 65, 89

Maxted, P. F. L., Hill, G., \& Hilditch, R. W. 1995, A\&A, 301, 135

McCook, G. 1971, AJ, 76, 449

Narita, E., Schröder, K. P., \& Smith, R. C. 2001, The Observatory, $121,308 \mathrm{~N}$

Popper, D. M. 1980, ARA\&A, 18, 115

Popper, D. M. 1989, ApJS, 71, 596

Press, W. H., Teukolsky, S. A., Vetterling, W. T., \& Flannery, B. P. 1992, Numerical Recipes in Fortran, The Art of Scientific Computing, Second Edition (New York: Cambridge University Press), 120

Rafert, J. B., \& Twigg, L. W. 1980, MNRAS, 193, 79

Rucinski, S. M. 1969, Acta Astr., 19, 245

Russo, G., \& Milano, L. 1983, A\&AS, 52, 311

Struve, O. 1950, ApJ, 112, 184

Tomkin, J., \& Lambert, D. L. 1978, ApJ, 222, L119

Vivekananda Rao, P., \& Sarma, M. B. K. 1997, Bull. Astr. Soc. India, 25,93

Wilson, R. E., \& Devinney, E. J. 1971, ApJ, 166, 605

Zeipel, H. V. 1924, MNRAS, 84, 702 